\title{
Decreasing Hypoglycemia following Insulin Administration for Inpatient Hyperkalemia
}

\author{
Allen Vinh Tran, PharmD ${ }^{1}$, Robert J Rushakoff, MD², Priya Prasad, $\mathrm{PhD}^{3}$, \\ Sara G Murray, MD, MAS ${ }^{3}$, Bradley Monash, MD ${ }^{3}$, Heidemarie Macmaster, PharmD ${ }^{4}$
}

${ }^{1}$ School of Pharmacy, University of California, San Francisco, California; ${ }^{2}$ Division of Endocrinology and Metabolism, University of California, San Francisco, California; ${ }^{3}$ Division of Hospital Medicine, University of California, San Francisco, California; ${ }^{4}$ Institute for Nursing Excellence, University of California, San Francisco, California, (currently at Lahey Health System, Burlington, Massachusetts).

BACKGROUND: Acute hyperkalemia (serum potassium $\geq$ $5.1 \mathrm{mEq} / \mathrm{L}$ ) is often treated with a bolus of IV insulin. This treatment may result in iatrogenic hypoglycemia (glucose $<70 \mathrm{mg} / \mathrm{dll}$ ).

OBJECTIVES: The aims of this study were to accurately determine the frequency of iatrogenic hypoglycemia following insulin treatment for hyperkalemia, and to develop an electronic health record (EHR) orderset to decrease the risk for iatrogenic hypoglycemia.

DESIGN: This study was an observational, prospective study.

SETTING: The setting for this study was a university hospital.

PATIENTS: All nonobstetric adult inpatients in all acute and intensive care units were eligible.

INTERVENTION: Implementation of a hyperkalemia orderset (Orderset 1.1) with glucose checks before and then one, two, four, and six hours after regular intravenous insulin administration. Based on the results from Orderset 1.1, Orderset 1.2 was developed and introduced to include weight-based dosing of insulin options, alerts identifying patients at higher risk of hypoglycemia, and tools to guide decision-making based on the preinsulin blood glucose level.

MEASUREMENTS: Patient demographics, weight, diabetes history, potassium level, renal function, and glucose levels were recorded before, and then glucose levels were measured again at one, two, four, and six hours after insulin was administered.

RESULTS: The iatrogenic hypoglycemia rate identified with mandatory glucose checks in Orderset 1.1 was $21 \%$; $92 \%$ of these occurred within three hours posttreatment. Risk factors for hypoglycemia included decreased renal function (serum creatinine $>2.5 \mathrm{mg} / \mathrm{dl}$ ), a high dose of insulin $(>0.14$ units $/ \mathrm{kg})$, and re-treatment with blood glucose $<140 \mathrm{mg} / \mathrm{dl}$. After the introduction of Orderset 1.2 , the rate of iatrogenic hypoglycemia decreased to $10 \%$.

CONCLUSIONS: The use of an EHR orderset for treating hyperkalemia may reduce the risk of iatrogenic hypoglycemia in patients receiving insulin while still adequately lowering their potassium. Journal of Hospital Medicine 2020;15:81-86. () 2020 Society of Hospital Medicine yperkalemia (serum potassium $\geq 5.1 \mathrm{mEq} / \mathrm{L}$ ), if left untreated, may result in cardiac arrhythmias, severe muscle weakness, or paralysis. ${ }^{1,2}$ Insulin administration can rapidly correct hyperkalemia by shifting serum potassium intracellularly. ${ }^{3}$ Treatment of hyperkalemia with insulin may lead to hypoglycemia, which, when severe, can cause confusion, seizures, loss of consciousness, and death. The use of regular and short-acting insulins to correct hyperkalemia quickly in hospitalized patients results in the greatest risk of hypoglycemia within three hours of treatment. ${ }^{4}$ Nonetheless, monitoring blood glucose levels within six hours of

*Corresponding Author: Robert J. Rushakoff, MD; E-mail: robert.rushakoff@ ucsf.edu; Telephone: 415-885-3868

Find additional supporting information in the online version of this article.

Received: July 28, 2019; Revised: October 18, 2019;

Accepted: November 10, 2019

(C) 2020 Society of Hospital Medicine DOI 10.12788/jhm.3357 postinsulin administration is not a standard part of hyperkalemia treatment guidelines, ${ }^{3}$ leaving the rates of hypoglycemia in this setting poorly characterized.

Without standardized blood glucose measurement protocols, retrospective studies have reported posttreatment hypoglycemia rates of $8.7 \%-17.5 \%$ among all patients with hyperkalemia, 5,6 and $13 \%$ among patients with end-stage renal disease. ${ }^{4}$ These estimates likely underestimate the true hypoglycemia rates as they measure blood glucose sporadically and are often outside the three-hour window of highest risk after insulin administration.

At the University of California, San Francisco Medical Center (UCSFMC), we faced similar issues in measuring the true hypoglycemia rates associated with hyperkalemia treatment. In December 2015, a 12-month retrospective review revealed a $12 \%$ hypoglycemia rate among patients treated with insulin for hyperkalemia. This review was limited by the inclusion of only patients treated for hyperkalemia using the standard orderset supplied with the electronic health record system (EHR; 
EPIC Systems, Verona, Wisconsin) and the absence of specific orders for glucose monitoring. As a result, more than $40 \%$ of these inpatients had no documented glucose within six hours of postinsulin administration.

We subsequently designed and implemented an adult inpatient hyperkalemia treatment orderset aimed at reducing iatrogenic hypoglycemia by promoting appropriate insulin use and blood glucose monitoring during the treatment of hyperkalemia. Through rapid improvement cycles, we iteratively revised the orderset to optimally mitigate the risk of hypoglycemia that was associated with insulin use. We describe implementation and outcomes of weight-based insulin dosing, ${ }_{1}^{7}$ automated alerts to identify patients at greatest risk for hypoglycemia, and clinical decision support based on the preinsulin blood glucose level. We report the rates of iatrogenic hypoglycemia after the implementation of these order-set changes.

\section{METHODS}

\section{Design Overview}

EHR data were extracted from Epic Clarity. We analyzed data following Orderset 1.1 implementation (January 1, 2016-March $19,2017)$ when hypoglycemia rates were reliably quantifiable and following orderset revision 1.2 (March 20, 2017-September $30,2017)$ to evaluate the impact of the orderset intervention. The data collection was approved by the Institutional Review Board at the University of California, San Francisco.

Additionally, we explored the frequency in which providers ordered insulin through the hyperkalemia orderset for each version of the orderset via two-month baseline reviews. Investigation for Orderset 1.1 was from January 1, 2017 to February 28, 2017 and for Orderset 1.2 was from August 1, 2017 to September 30, 2017. Insulin ordering frequency through the hyperkalemia orderset was defined as ordering insulin through the adult inpatient hyperkalemia orderset versus ordering insulin in and outside of the hyperkalemia orderset.

Last, we measured the nursing point of care testing (POCT) blood glucose measurement compliance with the hyperkalemia orderset. Nursing utilization acceptance of the hyperkalemia orderset was defined as adequate POCT blood glucose levels monitored in comparison to all insulin treatments via the hyperkalemia orderset.

\section{Setting and Participants}

We evaluated nonobstetric adult inpatients admitted to UCSF Medical Center between January 2016 and September 2017. All medical and surgical wards and intensive care units were included in the analysis.

\section{Intervention}

In June 2012, an EHR developed by Epic Systems was introduced at UCSFMC. In January 2016, we designed a new EHRbased hyperkalemia treatment orderset (Orderset 1.1), which added standard POCT blood glucose checks before and at one, two, four, and six hours after insulin injection (Appendix 1). In March 2017, a newly designed orderset (Orderset 1.2) replaced the previous hyperkalemia treatment orderset $\mathrm{Ap}$ - pendix 2). Orderset 1.2 included three updates. First, providers were now presented the option of ordering insulin as a weightbased dose ( 0.1 units $/ \mathrm{kg}$ intravenous bolus of regular insulin) instead of the previously standard 10 units. Next, provider alerts identifying high-risk patients were built into the EHR. Last, the orderset included tools to guide decision-making based on the preinsulin blood glucose as follows: (1) If preinsulin blood glucose is less than $150 \mathrm{mg} / \mathrm{dL}$, then add an additional dextrose $50 \%$ (50 mL) IV once one hour postinsulin administration, and (2) if preinsulin blood glucose is greater than $300 \mathrm{mg} / \mathrm{dL}$, then remove dextrose $50 \%(50 \mathrm{~mL})$ with insulin administration.

Inclusion and exclusion criteria are shown in the Figure. All patients who had insulin ordered via a hyperkalemia orderset were included in an intention-to-treat analysis. A further analysis was performed for patients for whom orderset compliance was achieved (ie, insulin ordered through the ordersets with adequate blood glucose monitoring). These patients were required to have a POCT blood glucose check preinsulin administration and postinsulin administration as follows: (1) between 30 to 180 minutes ( 0.5 to three hours) after insulin administration, and (2) between 180 and 360 minutes (three to six hours) after insulin administration. For patients receiving repeated insulin treatments for hyperkalemia within six hours, the first treatment data points were excluded to prevent duplication.

\section{Outcomes}

We extracted data on all nonobstetric adult patients admitted to UCSFMC between January 1, 2016 and March 19, 2017 (Orderset 1.1) and between March 20, 2017 and September 30, 2017 (Orderset 1.2).

We measured unique insulin administrations given that each insulin injection poses a risk of iatrogenic hypoglycemia. Hypoglycemia was defined as glucose $<70 \mathrm{mg} / \mathrm{dL}$ and severe hypoglycemia was defined as glucose $<40 \mathrm{mg} / \mathrm{dL}$. Covariates included time and date of insulin administration; blood glucose levels before and at one, two, four, and six hours after insulin injection (if available); sex; weight; dose of insulin given for hyperkalemia treatment; creatinine; known diagnosis of diabetes; concomitant use of albuterol; and concomitant use of corticosteroids. Hyperglycemia was defined as glucose $>180 \mathrm{mg} / \mathrm{dL}$. We collected potassium levels pre- and postinsulin treatment. The responsible team's discipline and the location of the patient (eg, medical/surgical unit, intensive care unit, emergency department) where the insulin orderset was used were recorded.

\section{Statistical Analysis}

Statistical analysis for our data included the $\chi 2$ test for categorical data and Student $t$ test for continuous data. The bivariate analysis identified potential risk factors and protective factors for hypoglycemia, and logistic regression was used to determine independent predictors of hypoglycemia. Through bivariate analyses, any factor with a $P$ value below .05 was included in the multivariable analyses to investigate a significant contribution to hypoglycemia outcomes. Analyses for hypoglycemia and severe hypoglycemia rates, potassium levels pre- and postinsulin treatment, and hyperglycemia rates were done for 
Orderset $1.1(1 / 1 / 16-3 / 19 / 17)$

Features of the Orderset

1. Hyperkalemia Treatment Modalities

- Insulin 10 Units IV x1

- D50 25g IV x1

2. POCT Pre- and Post-Insulin Administration

352 Insulin Orders via Hyperkalemia Orderset

15 Orders Excluded for Repeat Treatment

337 Remaining Insulin Orders

112 Orders Excluded for Inadequate POCT

- Lack in Pre-BG

- Lack in Post-BG First 3 hours

- Lack in Post-BG Latter 3 hours

$\rightarrow \quad \mathrm{N}=225$

\section{Orderset $1.2(3 / 20 / 17-9 / 30 / 17)$}

Features of the Orderset

1. Hyperkalemia Treatment Modalities

2. POCT Pre- and Post-Insulin Administration

3. Weight-Based Dosing for Insulin (0.1 units/kg)-NEW

4. Provider alerts to Identofy At-Risk Patients for Hypoglycemia NEW

5. Concomitant Dextrose Administration Based on Pre-Insulin Blood Glucose - NEW

-If Pre-Insulin Blood Glucose $<150 \mathrm{mg} / \mathrm{dL}$, then Add an Additional Dextrose 50\% (25g) IV Once 1-Hour Post-Insulin Admonistration.

-If Pre-Insulin BLood Glucose is $>300 \mathrm{mg} / \mathrm{dL}$, then Dextrose $50 \%(25 \mathrm{~g})$ with Insulin Administration.

239 Insulin Orders via Hyperkalemia Orderset

14 Orders Excluded for Repeat Treatment

225 Remaining Insulin Orders

80 Orders Excluded for Inadequate POCT

- Lack in Pre-BG

- Lack in Post-BG First 3 hours

- Lack in Post-BG Latter 3 hours

$N=145$
FIG. Flow Diagram Displaying the Study Cohort's Inclusion and Exclusion Criteria Abbreviation: POCT, point of care testing.

both the intention-to-treat group and the group with all criteria met. All analyses were rendered utilizing Stata version 14 (Stata Corp LLC, College Station, Texas).

\section{RESULTS}

Baseline patient characteristics, initial insulin dosing, the treatment team, and the location are shown in Table 1. With the implementation of weight-based dosing, a lower dose of insulin was administered with Orderset 1.2 compared with Orderset 1.1.

Orderset adherence rates for Orderset 1.1 and 1.2 were as follows: Acute Care Floor 65\% (70\%), Intensive Care Unit 63\% (66\%), and Emergency Department 60\% (55\%). A two-month audit of orderset usage and compliance revealed that 73\% (70 of 96) of insulin treatments were ordered through Orderset 1.1, and $77 \%$ (71 of 92) of insulin treatments were ordered through Orderset 1.2. The distribution of orderset usage across location and primary service are shown in Table 1.
The patient distribution is shown in the Figure. In the Orderset 1.1 period, there were 352 total insulin treatments utilizing the newly revised UCSFMC adult inpatient hyperkalemia orderset that were used for the intention-to-treat analysis, and there were 225 patients for whom compliance with orderset monitoring was achieved. Notably, 112 treatments were excluded for the lack of adequate blood glucose monitoring. In the Orderset 1.2 period, there were 239 total insulin treatments utilizing the newly revised UCSFMC adult inpatient hyperkalemia orderset that were used for the intention-to-treat analysis, and there were 145 patients for whom compliance with orderset monitoring was achieved. During this phase, 80 treatments were excluded for inadequate blood glucose monitoring.

Predictors of hypoglycemia following the implementation of Orderset 1.1 are shown in Table 2, and the logistic regression model of these risks is shown in Appendix Table 1. Female gender, weight-based dosing of insulin exceeding 0.14 units/ 
TABLE 1. Baseline Characteristics, Initial Insulin Dosing, and Treatment Team and Location ${ }^{a}$

\begin{tabular}{|c|c|c|c|}
\hline & $\begin{array}{c}\text { Orderset } 1.1 \\
n=225\end{array}$ & $\begin{array}{c}\text { Orderset } 1.2 \\
n=145\end{array}$ & $P$ Value \\
\hline Age (y) & $58.6(14.4)$ & $56.9(17.0)$ & .372 \\
\hline Female & $78(35 \%)$ & $60(41 \%)$ & .245 \\
\hline Weight (kg) & $78.8(26.9)$ & $75.7(25.4)$ & .349 \\
\hline Weight-Based Dose (units/kg) & $0.12(0.05)$ & $0.09(0.02)$ & $<.0001$ \\
\hline Preinsulin Glucose (mg/dL) & $170(140)$ & $173(114)$ & .806 \\
\hline Serum Creatinine (mg/dL) & $4.22(3.64)$ & $4.39(3.79)$ & .714 \\
\hline Type 2 Diabetes & $77(34 \%)$ & $54(37 \%)$ & .614 \\
\hline Concomitant Albuterol & $72(32 \%)$ & $39(27 \%)$ & .298 \\
\hline Concomitant Corticosteroid & $14(6 \%)$ & $9(6 \%)$ & .995 \\
\hline Location & & & $.083^{c}$ \\
\hline Intensive Care Unit & $87(39 \%)$ & $41(28 \%)$ & \\
\hline Medical/Surgical Unit & $82(36 \%)$ & $56(39 \%)$ & \\
\hline Emergency Department & $56(25 \%)$ & $48(33 \%)$ & \\
\hline Primary Service & & & $.111^{c}$ \\
\hline Surgery & $62(28 \%)$ & $26(18 \%)$ & \\
\hline Medicine Services ${ }^{\mathrm{b}}$ & $61(27 \%)$ & $37(26 \%)$ & \\
\hline Transplant & $46(20 \%)$ & $34(23 \%)$ & \\
\hline Emergency Department & $56(25 \%)$ & $48(33 \%)$ & \\
\hline
\end{tabular}

Values Expressed as Mean (Standard Deviation) or Number (\%)

a Data presented for patients for whom compliance with orderset glucose monitoring was achieved

${ }^{b}$ General and Subspecialty Medicine Services

${ }^{c} P$ value from chi-squared test for location and primary service

$\mathrm{kg}$, preinsulin blood glucose less than $140 \mathrm{mg} / \mathrm{dL}$, and serum creatinine greater than $2.5 \mathrm{mg} / \mathrm{dl}$ were associated with an increased risk of hypoglycemia. A known diagnosis of Type 2 diabetes, concomitant albuterol within one hour of insulin administration, and corticosteroid administration within two hours of insulin administration were associated with a decreased risk of hypoglycemia.

The rates of hypoglycemia $(<70 \mathrm{mg} / \mathrm{dl})$ and severe hypoglycemia ( $<40 \mathrm{mg} / \mathrm{dl})$ are shown in Table 3. During the Orderset 1.1 period, for patients with all criteria met, 48 of $225(21 \%)$ had hypoglycemia, and 11 of 225 (5\%) had severe hypoglycemia. In the first three hours after insulin administration, 92\% (44/48) of these hypoglycemic events occurred, with the remaining hypoglycemic events $(8 \%, 4 / 48)$ occurring in the last three hours.

During the Orderset 1.2 period, for patients with all criteria met, 14 of 145 (10\%) had hypoglycemia, and three of 145 (2\%) had severe hypoglycemia. Ten of 14 (72\%) of these hypoglycemic events occurred in the first three hours, with the remaining four hypoglycemic events (28\%) occurring in the last three hours.

An intention-to-treat analysis for hyperglycemia, defined as glucose $>180 \mathrm{mg} / \mathrm{dl}$, revealed that during the Orderset $1.1 \mathrm{pe}$ riod, 80 of 352 (23\%) had hyperglycemia before insulin administration, and 38 of $352(11 \%)$ had hyperglycemia after insulin administration. During the Orderset 1.2 period, 52 of 239 (22\%) had hyperglycemia before insulin administration, and 15 of 239 (6\%) had hyperglycemia after insulin administration. Results can be found in Table 3.

Pre- and posttreatment potassium levels are shown in Table 3. An intention-to-treat analysis for potassium reduction postinsulin administration revealed that during the Orderset 1.1 period, there was an absolute reduction of $0.73 \mathrm{mmol} / \mathrm{L}$, while during the Orderset 1.2 period, there was an absolute reduction of $0.95 \mathrm{mmol} / \mathrm{L}$.

\section{DISCUSSION}

Treatment of hyperkalemia with insulin may result in significant iatrogenic hypoglycemia. Prior studies have likely underestimated the incidence of hyperkalemia treatment-associated hypoglycemia as glucose levels are rarely checked within three hours of insulin administration. ${ }^{8}$ In our study, which was designed to ensure appropriate blood glucose measurement, $21 \%$ of insulin treatments for hyperkalemia resulted in hypoglycemia, with $92 \%$ of hypoglycemic events occurring within the first three hours.

For the Orderset 1.1 period, patient risk factors identified for iatrogenic hypoglycemia postinsulin administration were female sex, doses of regular insulin greater than 0.14 units $/ \mathrm{kg}$, prein- 
TABLE 2. Predictors of Hypoglycemia following Implementation of Orderset 1.1

\begin{tabular}{|c|c|c|c|}
\hline Orderset 1.1 & $\begin{array}{l}\text { Treatments without } \\
\text { Hypoglycemia } \\
(n=177)\end{array}$ & $\begin{array}{l}\text { Treatments with } \\
\text { Hypoglycemia } \\
\quad(n=48)\end{array}$ & $P$ Value \\
\hline Female $e^{(r)}$ & $67(38 \%)$ & $11(23 \%)$ & .070 \\
\hline$>0.14$ Units/kg Insulin & $48(27 \%)$ & $24(50 \%)$ & .005 \\
\hline Preinsulin Blood Glucose $<140 \mathrm{mg} / \mathrm{dL}^{(r)}$ & $79(45 \%)$ & $41(85 \%)$ & $<.0001$ \\
\hline Serum Creatinine $>2.5 \mathrm{mg} / \mathrm{dL}^{(t)}$ & $87(49 \%)$ & $32(67 \%)$ & .040 \\
\hline Diagnosed with Type 2 Diabetes ${ }^{(p)}$ & $67(87 \%)$ & $10(13 \%)$ & .039 \\
\hline Concomitant Albuterol(p) & $49(28 \%)$ & $23(48 \%)$ & .008 \\
\hline Concomitant Corticosteroid ${ }^{(\rho)}$ & $11(6 \%)$ & $3(6 \%)$ & .993 \\
\hline
\end{tabular}

Risk Factors ${ }^{(r)}$; Protective Factors ${ }^{(p)}$

TABLE 3. Rates of Hypoglycemia, Hyperglycemia, and Potassium Levels with Ordersets 1.1 and 1.2

\begin{tabular}{|c|c|c|c|}
\hline & Orderset 1.1 & Orderset 1.2 & $P$ Value \\
\hline \multicolumn{4}{|l|}{ Hypoglycemia } \\
\hline All Criteria Met (n) & 225 & 145 & \\
\hline Glucose $<70 \mathrm{mg} / \mathrm{dl}^{\mathrm{a}}$ & $48(21 \%)$ & $14(10 \%)$ & $<.008$ \\
\hline Glucose $<40 \mathrm{mg} / \mathrm{dl}^{\mathrm{a}}$ & $11(5 \%)$ & $3(2 \%)$ & .207 \\
\hline Intention to Treat (n) & 352 & 239 & \\
\hline Glucose $<70 \mathrm{mg} / \mathrm{dl}^{\mathrm{a}}$ & $56(16 \%)$ & $19(8 \%)$ & $<.006$ \\
\hline Glucose $<40 \mathrm{mg} / \mathrm{dl}^{a}$ & $14(4) \%$ & $5(2 \%)$ & .149 \\
\hline \multicolumn{4}{|l|}{ Hyperglycemia (glucose > $180 \mathrm{mg} / \mathrm{dl}$ ) } \\
\hline \multicolumn{4}{|l|}{ Intention to Treat } \\
\hline Pretreatment $^{\mathrm{a}}$ & $81(23 \%)$ & $53(22 \%)$ & .781 \\
\hline Posttreatment $^{\mathrm{a}}$ & $39(11 \%)$ & $14(6 \%)$ & .059 \\
\hline \multicolumn{4}{|l|}{ Potassium Levels (mEq/L) } \\
\hline \multicolumn{4}{|l|}{ Intention to Treat } \\
\hline Pretreatment potassium ${ }^{b}$ & $6.12(0.71)$ & $6.23(0.83)$ & .092 \\
\hline Posttreatment Potassium ${ }^{b}$ & $5.39(0.94)$ & $5.27(0.85)$ & .125 \\
\hline Potassium Reduction Posttreatment ${ }^{b}$ & $0.73(0.85)$ & $0.95(0.80)$ & .002 \\
\hline
\end{tabular}

sulin blood glucose less than $140 \mathrm{mg} / \mathrm{dL}$, and serum creatinine greater than $2.5 \mathrm{mg} / \mathrm{dL}$. These results are consistent with studies suggesting that preinsulin blood glucose levels less than 140 $\mathrm{mg} / \mathrm{dL}$ and the standard 10 units of insulin for hyperkalemia treatment may increase the risk of hypoglycemia. ${ }^{4,7,9}$

To decrease the risk of iatrogenic hypoglycemia, we redesigned our hyperkalemia insulin orderset to address the strongest predictors of hypoglycemia (doses of regular insulin greater than 0.14 units $/ \mathrm{kg}$ and preinsulin blood glucose less than 140 $\mathrm{mg} / \mathrm{dL}$ ). The main changes were weight-based insulin dosing (based on previously published data) ${ }^{10}$ and adjustment of glu- cose administration based on the patient's glucose levels. ${ }^{11}$ Following these changes, the rates of both hypoglycemia and severe hypoglycemia were statistically significantly reduced. In addition, of the 14 hypoglycemia events identified after the introduction of Orderset 1.2, five could have been prevented (36\%) had the protocol been strictly followed. These five hypoglycemia events occurred later than one-hour postinsulin administration in patients with blood sugars $<150 \mathrm{mg} / \mathrm{dL}$ prior to insulin administration. In each of these cases, Orderset 1.2 called for an additional dextrose $50 \%(50 \mathrm{~mL})$ IV bolus, which likely would have prevented the subsequently recorded hypo- 
glycemia. In other words, our orderset indicated that these patients received an additional bolus of dextrose. However, they did not receive their glucose at the appropriate time, contributing to the hypoglycemia events. The orderset did not include a best practice alert (BPA) to remind providers about the extra dextrose bolus. In the future, we plan to add this BPA.

The hypoglycemia rate identified by Orderset 1.1 was $21 \%$ and the hypoglycemia rate identified by the Orderset 1.2 was $10 \%$. The severe hypoglycemia rate identified by Orderset 1.1 was $5 \%$ and the severe hypoglycemia rate identified by Orderset 1.2 was $2 \%$. The hypoglycemia and severe hypoglycemia rates significantly decreased after the introduction of Orderset 1.2. To mimic a real-world clinical setting, where monitoring of blood glucose is not always achieved multiple times within a six-hour timeframe of postinsulin treatment for hyperkalemia, we conducted an intention-to-treat analysis. Even when including patients for whom full blood glucose monitoring was not achieved, the introduction of Orderset 1.2 was associated with a significant decrease in the hypoglycemia rate.

To demonstrate whether weight-based dosing of insulin was as effective as a standard dose for hyperkalemia treatment, we compared the impact of Orderset 1.1, which only had the option for single standard doses of insulin, with the impact of Orderset 1.2, which included weight-based dosing options. With the introduction of Orderset 1.2, there was a significant decrease in serum potassium, indicating that weight-based dosing options may not only prevent hypoglycemia but may potentially provide more effective hyperkalemia treatment.

We also compared the rate of hyperglycemia (a glucose $>180 \mathrm{mg} / \mathrm{dl}$ ) pre- and posttreatment (Table 3). Although not statistically significant, the rate of hyperglycemia decreased from $11 \%$ to $6 \%$, suggesting a trend toward decreased hyperglycemia with orderset usage.

As orderset usage for hyperkalemia management only occurred approximately $75 \%$ of the time, likely, forcing the use of these ordersets would further reduce the incidence of treatment-associated hypoglycemia. To encourage the use of ordersets for hyperkalemia management, our medical center has largely restricted insulin ordering so that it can only be done through ordersets with the proper precautions in place, regardless of the indication. Furthermore, adherence to all the blood glucose monitoring orders embedded in the ordersets remained suboptimal irrespective of managing the service or clinical setting. While we believe that $100 \%$ of postglucose monitoring should be possible with appropriate education and institutional support, in some clinical environments, checking glucose levels at least twice in a six-hour window (postinsulin treatment) might be prohibitive. Since $92 \%$ of hypoglycemic events occurred within the first three hours postinsulin administration, checking glucose prior to insulin administration and within the first four hours following insulin administration should be prioritized.

Finally, development and implementation of these hyperkalemia treatment ordersets required an experienced multidisciplinary team, including pharmacists, nurses, hospitalists, endocrinologists, and EHR system programmers. ${ }^{12,13} \mathrm{We}$, therefore, encourage interprofessional collaboration for any institutions seeking to establish innovative clinical protocols.

This analysis was limited to the insulin administration meeting our inclusion criteria. Thus, we could not identify a true hypoglycemia rate for treatments that were not followed by adequate blood glucose monitoring postinsulin administration, or for insulin administration ordered outside of the hyperkalemia ordersets.

\section{CONCLUSION}

The use of a comprehensive EHR orderset for the treatment of hyperkalemia with predefined times for blood glucose monitoring, weight-based insulin dosing, and prompts to warn providers of an individual patient's risk for hypoglycemia may significantly reduce the incidence of iatrogenic hypoglycemia.

Disclosures: Dr. Prasad serves as a paid consulting epidemiologist for EpiExcellence,LLC, outside the submitted work. All other authors have nothing to disclose.

\section{References}

1. Acker CG, Johnson JP, Palevsky PM, Greenberg A. Hyperkalemia in hospitalized patients: causes, adequacy of treatment, and results of an attempt to improve physician compliance with published therapy guidelines. Arch Intern Med. 1998;158(8):917-924. https://doi.org/10.1001/archinte.158.8.917.

2. Fordjour KN, Walton T, Doran JJ. Management of hyperkalemia in hospitalized patients. Am J Med Sci. 2014;347(2):93-100. https://doi.org/10.1097/ MAJ.0b013e318279b105.

3. Part-10-Special-Circumstances-of-Resuscitation.pdf. https://eccguidelines.heart.org/wp-content/themes/eccstaging/dompdf-master/pdffiles/ part-10-special-circumstances-of-resuscitation.pdf. Accessed December 16, 2017.

4. Apel J, Reutrakul S, Baldwin D. Hypoglycemia in the treatment of hyperkalemia with insulin in patients with end-stage renal disease. Clin Kidney J. 2014;7(3):248-250. https://doi.org/10.1093/ckj/sfu026.

5. Schafers S, Naunheim R, Vijayan A, Tobin G. Incidence of hypoglycemia following insulin-based acute stabilization of hyperkalemia treatment. J Hosp Med. 2012;7(3):239-242. https://doi.org/10.1002/jhm.977

6. Boughton CK, Dixon D, Goble E, et al. Preventing hypoglycemia following treatment of hyperkalemia in hospitalized patients. J Hosp Med. 2019;14:E1E4. https://doi.org/10.12788/jhm.3145.

7. Wheeler DT, Schafers SJ, Horwedel TA, Deal EN, Tobin GS. Weight-based insulin dosing for acute hyperkalemia results in less hypoglycemia. J Hosp Med. 2016;11(5):355-357. https://doi.org/10.1002/jhm.2545

8. Coca A, Valencia AL, Bustamante J, Mendiluce A, Floege J. Hypoglycemia following intravenous insulin plus glucose for hyperkalemia in patients with impaired renal function. PLoS ONE. 2017;12(2):e0172961. https://doi. org/10.1371/journal.pone.0172961.

9. LaRue HA, Peksa GD, Shah SC. A comparison of insulin doses for the treatment of hyperkalemia in patients with renal insufficiency. Pharmacotherapy. 2017;37(12):1516-1522. https://doi.org/10.1002/phar.2038.

10. Brown K, Setji TL, Hale SL, et al. Assessing the impact of an order panel utilizing weight-based insulin and standardized monitoring of blood glucose for patients with hyperkalemia. Am J Med Qual. 2018;33(6):598-603. https:// doi.org/10.1177/1062860618764610.

11. Farina N, Anderson C. Impact of dextrose dose on hypoglycemia development following treatment of hyperkalemia. Ther Adv Drug Saf. 2018;9(6):323329. https://doi.org/10.1177/2042098618768725.

12. Neinstein A, MacMaster HW, Sullivan MM, Rushakoff R. A detailed description of the implementation of inpatient insulin orders with a commercial electronic health record system. J Diabetes Sci Technol. 2014;8(4):641-651. https://doi.org/10.1177/1932296814536290.

13. MacMaster HW, Gonzalez S, Maruoka A, et al. Development and implementation of a subcutaneous Insulin pen label bar code scanning protocol to prevent wrong-patient insulin pen errors. Jt Comm J Qual Patient Saf. 2019;45(5):380-386. https://doi.org/10.1016/j.jcjq.2018.08.006. 